\title{
On optimal credibility premiums in multiperiod insurance
}

ARTICLE in APPLICATIONES MATHEMATICAE · JANUARY 2014

DOI: $10.4064 / a m 41-1-3$

READS

16

2 AUTHORS, INCLUDING:

Marek Kaluszka

Lodz University of Technology

71 PUBLICATIONS 296 CITATIONS

SEE PROFILE 


\title{
ON OPTIMAL CREDIBILITY PREMIUMS IN MULTIPERIOD INSURANCE
}

\author{
Wojciech Antoniak, Marek Kałuszka
}

\begin{abstract}
This paper focuses on the problem of an optimal stream of premiums in a multiperiod credibility model. Formulas are derived for given claim history (screening) and individual information unknown for insurance company (signaling) but under the assumption that the coverage period is not fixed because of e.g. lapses, renewals, deaths, total losses etc. It is shown that the derived stream reflects better the corresponding risk than other approaches.
\end{abstract}

\section{INTRODUCTION}

The credibility theory is based on the assumption that each policyholder belongs to predefined class of risk characterized by a risk profile $\theta$, which is unknown for the insurance company. Let us consider the client, who generates during the period $t$ a claim modeled by random variable $X_{t}$. Let $f(\cdot \mid \theta)$ will be a conditional probability density of random variable $X_{t}$ when $\Theta=\theta$, with respect to some $\sigma$-finite measure. In the presented paper $f$ is independent from $t$ and random variable $\Theta$ is described by compound probability distribution $\pi(\theta)$. Let us assume that $\operatorname{Cov}\left(X_{i}, X_{j} \mid \Theta=\theta\right)=0$ for $i \neq j$, and for simplicity we put $\mu(\theta)=\mathrm{E}\left(X_{i} \mid \Theta=\theta\right)$ and $\sigma^{2}(\theta)=\operatorname{Var}\left(X_{i} \mid \Theta=\theta\right)$ for all $i$. Setting $m=\mathrm{E} \mu(\Theta), s^{2}=\mathrm{E} \sigma^{2}(\Theta)$ and $a^{2}=\operatorname{Var} \mu(\Theta)$, we assume that based on claim history the insurer knows exact value of coefficients $m, s^{2}$ and $a^{2}$. The main problem of insurance company at time $n$ is to valuate adequate net premium $P_{n+1}$ for a given client. The pricing process should take in the consideration claims $X_{1}, \ldots, X_{n}$ resulting respectively from periods $1, \ldots, n$. If an insurer knew the client's class of risk $\theta$, then the premium would be settled as $\widehat{P}_{n+1}=\mathrm{E}\left(X_{n+1} \mid \Theta=\theta\right)$. Other solution of this problem is to use an optimal linear predictor $P_{n+1}^{*}=\mathrm{E}\left(X_{n+1} \mid X_{1}, \ldots, X_{n}\right)$ where $P_{1}^{*}=\mathrm{E} X_{1}=m$. Unfortunately, in order to derive explicitly formula of premium $P_{n+1}^{*}$ it is essential to posses exact conditional distribution functions, which are known only in some cases, such as exponential models. Bühlmann proposed that premium should be set as an linear predictor of random variable $X_{n+1}$, 
i.e., the predictor $P_{n+1}=a_{0}+\sum_{i=1}^{n} a_{i} X_{i}$, which minimalizes mean squared error $\mathrm{E}\left(P_{n+1}-X_{n+1}\right)^{2}$, where $P_{1}=\mathrm{E} X_{1}$. The solution of such settled problem is credibility premium

$$
P_{n+1}^{C r}=z_{n} \bar{X}_{n}+\left(1-z_{n}\right) m
$$

where $\bar{X}_{n}=\left(X_{1}+\ldots+X_{n}\right) / n$ and

$$
z_{n}=\frac{a^{2} n}{s^{2}+a^{2} n}
$$

is credibility coefficient (see [1], [2], [3] and [6])).

In the last years credibility premium was thoroughly investigated. Various changes were proposed, which extended or adjusted the Bühl-

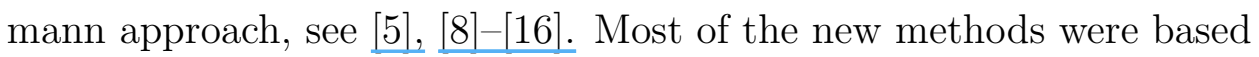
on modifications of loss function.

In this paper we propose a new method of premium calculation. Our enhancement is based on the assumption that some clients know their class of risk. We show that the new premium correspond better to future losses. Our considerations begins with one period insurance contract. Obtained results are applied to multiperiod insurance contracts.

\section{ONE PERIOD MODEL}

The generalization of the Bühlmann model, on the purpose of an application use, is the Bühlmann-Straub model. Its construction begins with setting independent random vectors

$$
\left(X_{i, 1}, \ldots, X_{i, n_{i}}, X_{i, n_{i}+1}\right), \quad i=1,2, \ldots, N
$$

which describe losses generated by one ( $i$ th) of the $N$ clients. They can belong to different classes of risks specified by risk profiles $\theta$. Let us assume that risk profile of the $i$ th client, characterized by random variable $\Theta_{i}$, is unknown for insurer, where $\Theta_{1}, \ldots, \Theta_{N}$ is a finite sequence of independent identically distributed random variables. Furthermore let us assume for all $i$ and $s \neq t$ that

$$
\begin{gathered}
\mathrm{E}\left(X_{i, t} \mid \Theta_{i}=\theta_{i}\right)=\mu\left(\theta_{i}\right), \quad \operatorname{Var}\left(X_{i, t} \mid \Theta_{i}=\theta_{i}\right)=\frac{\sigma^{2}\left(\theta_{i}\right)}{w_{i, t}}, \\
\operatorname{Cov}\left(X_{i, t}, X_{i, s} \mid \Theta_{i}=\theta\right)=0
\end{gathered}
$$


where $\mu$ i $\sigma$ are some functions, $w_{i, t}$ are known weights. In this paper we assume that insurer posses information about following coefficients

$$
m=\mathrm{E} \mu\left(\Theta_{i}\right), \quad s^{2}=\sigma^{2}\left(\Theta_{i}\right), \quad a^{2}=\operatorname{Var} \mu\left(\Theta_{i}\right) .
$$

Let us set $P$ as a net premium for the $i$ th client, based on known claims $\left(X_{j, 1}, \ldots, X_{j, n_{j}}\right)$ where $j=1, \ldots, N$.

In our approach the insurer is minimalizing not only inadequacy of premium $P$ to $X_{i, n_{i}+1}$ but also average premium for given client to his average claim, i.e the optimal premium $P=a_{0}+\sum_{j=1}^{n_{i}} a_{j} X_{i, j}$, should minimalize the following function

$$
I_{i}=\mathrm{E}\left(P-X_{i, n_{i}+1}\right)^{2}+\gamma^{2} \mathrm{E}\left(\mathrm{E}\left(P \mid \Theta_{i}\right)-\mathrm{E}\left(X_{i, n_{i}+1} \mid \Theta_{i}\right)\right)^{2},
$$

where $\gamma \geq 0$ is a fixed number. In the premium formula claims $X_{t, j}$ for $t \neq i$ are not included, because of independence from $X_{i, j}$. The optimal coefficients related to them are equal to zero.

Our approach is similar to the Markowitz optimal portfolio selection in that case that both methods take into consideration the average individual inadequacy of premium. Coefficient $\gamma$ describes unknown information for insurance company information, which can be settled by client during the acquisition of the insurance policy (signaling).

First, note that

$$
\begin{aligned}
I_{i}= & \operatorname{Var}\left(\sum_{j=1}^{n_{i}} a_{j} X_{i, j}-X_{i, n_{i}+1}\right)+\left(a_{0}-\left(1-\sum_{j=1}^{n_{i}} a_{j}\right) m\right)^{2} \\
& +\gamma^{2}\left(a_{0}-\left(1-\sum_{j=1}^{n_{i}} a_{j}\right) m\right)^{2}+\gamma^{2}\left(1-\sum_{j=1}^{n_{i}} a_{j}\right)^{2} a^{2}
\end{aligned}
$$

Hence, the optimal coefficient is equal to

$$
\widehat{a}_{0}=\left(1-\sum_{j=1}^{n_{i}} a_{j}\right) m \text {. }
$$

Furthermore for all $i, j$ and $s \neq t$ we have

$$
\operatorname{Var} X_{i, j}=\operatorname{Var}\left(\mathrm{E}\left(X_{i, j} \mid \Theta_{i}\right)\right)+\mathrm{E} \operatorname{Var}\left(X_{i, j} \mid \Theta_{i}\right)=a^{2}+\frac{s^{2}}{w_{i, j}},
$$

$\operatorname{Cov}\left(X_{i, t}, X_{i, s}\right)=\operatorname{Cov}\left(\mathrm{E}\left(X_{i, t} \mid \Theta_{i}\right), \mathrm{E}\left(X_{i, s} \mid \Theta_{i}\right)\right)+\mathrm{E} \operatorname{Cov}\left(X_{i, t}, X_{i, s} \mid \Theta_{i}\right)=a^{2}$, 
which including equations (3) and (4) imply

$$
\begin{aligned}
\min _{\left(a_{j}\right)} I_{i}= & \sum_{t=1}^{n_{i}} \sum_{s=1}^{n_{i}} a_{t} a_{s} \operatorname{Cov}\left(X_{i, t}, X_{i, s}\right)-2 \operatorname{Cov}\left(\sum_{j=1}^{n_{i}} a_{j} X_{i, j}, X_{i, n_{i}+1}\right) \\
& +\operatorname{Var} X_{i, n_{i}+1}+\gamma^{2}\left(1-\sum_{j=1}^{n_{i}} a_{j}\right)^{2} a^{2} \\
= & \sum_{j=1}^{n_{i}} a_{j}^{2}\left(a^{2}+\frac{s^{2}}{w_{i, j}}\right)+\sum_{t \neq s} a_{s} a_{t} a^{2}-2 \sum_{j=1}^{n_{i}} a_{j} a^{2} \\
& +\left(a^{2}+\frac{s^{2}}{w_{i, n_{i}+1}}\right)+\gamma^{2}\left(1-\sum_{j=1}^{n_{i}} a_{j}\right)^{2} a^{2} \\
= & \left(\sum_{j=1}^{n_{i}} a_{j}\right)^{2} a^{2}+\sum_{j=1}^{n_{i}} a_{j}^{2} \frac{s^{2}}{w_{i, j}}-2 a^{2} \sum_{j=1}^{n_{i}} a_{j}+\gamma^{2}\left(1-\sum_{j=1}^{n_{i}} a_{j}\right)^{2} a^{2} \\
& \left.+a^{2}+\frac{s^{2}}{w_{i, n_{i}+1}}\right)^{2}\left(1+\gamma^{2}\right)+s^{2} \sum_{j=1}^{n_{i}} a_{j}^{2} \frac{1}{w_{i, j}}+\frac{s^{2}}{w_{i, n_{i}+1}} . \\
= & a^{2}\left(\sum_{j=1}^{n_{i}} a_{j}-1\right)^{2}
\end{aligned}
$$

Applying the Cauchy-Schwarz inequality we have

$$
\left(\sum_{j=1}^{n_{i}} a_{j}\right)^{2}=\left(\sum_{j=1}^{n_{i}} \frac{a_{j}}{\sqrt{w_{i, j}}} \sqrt{w_{i, j}}\right)^{2} \leq \sum_{j=1}^{n_{i}} \frac{a_{j}^{2}}{w_{i, j}} \sum_{j=1}^{n_{i}} w_{i, j}
$$

and equality holds if and only if there exists a constant $c$ such as $\forall_{j} a_{j}=$ $c w_{i, j}$. Thus,

$$
\min _{\left(a_{j}\right)} I_{i}=\min _{c \in \mathbb{R}}\left[a^{2}\left(c w_{i}-1\right)^{2}\left(1+\gamma^{2}\right)+s^{2} c^{2} w_{i}\right]+\frac{s^{2}}{w_{i, n_{i}+1}},
$$

where $w_{i}=\sum_{j=1}^{n_{i}} w_{i, j}$. The minimum value is attained when

$$
c=\frac{a^{2}\left(1+\gamma^{2}\right)}{a^{2}\left(1+\gamma^{2}\right) w_{i}+s^{2}} .
$$

Summarizing the optimal premium is given by the formula

$$
P=z \sum_{j=1}^{n_{i}} \frac{w_{i, j}}{w_{i}} X_{i, j}+(1-z) m
$$


where

$$
z=\frac{a^{2}\left(1+\gamma^{2}\right) w_{i}}{a^{2}\left(1+\gamma^{2}\right) w_{i}+s^{2}}
$$

As it is in the Bühlmann-Straub model we have that $P \rightarrow m$, when $a \rightarrow$ 0 and $P-\bar{X}_{n} \rightarrow 0$, when $s^{2} \rightarrow 0$. Furthermore, when $w_{i j}=1$ for all $i, j$, the premium $P$ converge to individual net premium $\mathrm{E}\left(X_{1} \mid \Theta=\theta\right)$, when $n \rightarrow \infty$, but converges accelerates when $\gamma$ rises. If client is conscious that he is good, then he can set bigger value of $\gamma$. The average premium $\mu(\theta)$ for good clients is smaller than $m$.

\section{Multiple PeRiod MOdeL}

For simplicity further consideration are limited to the Bühlmann model, but it can be easily adjusted to the Bühlmann-Straub model. Let us assume that random variable $X_{t}$ describes loss generated by the $i$ th client during the period $t$, where $t=1,2, \ldots$. The premium $P_{t}$ for insurance policy, which covers claims characterized by $X_{t}$ and paid at the end of the period is receivable at the beginning of the period $t$. The premium $P_{t}$ is derived taking into consideration claim history, which are losses $X_{1}, \ldots, X_{t-1}$, where $n \leq t<T$ and $T$ can be a random variable. Optimal stream of premiums $\left(P_{t}\right)$ is given by the formula

$$
P_{t}=a_{0, t}+\sum_{i=1}^{t-1} a_{i, t} X_{i}, \quad a_{i, j} \in \mathbb{R},
$$

where coefficients $\left(a_{j, t}\right)$ are set in such a way that following function is minimalized

$$
\mathrm{E}\left[\sum_{t=n}^{T}\left(\left(P_{t}-X_{t}\right)^{2}+\gamma_{t}^{2}\left(\mathrm{E}\left(X_{t} \mid \Theta\right)-\mathrm{E}\left(P_{t} \mid \Theta\right)\right)^{2}\right)\right],
$$

where $\left(\gamma_{t}\right)$ is a sequence of nonnegative numbers.

Let us assume that random variables $T$ and $\left(X_{i}\right)$ are independent, i.e., $T$ is the future life of an owner of the insured real estate. Hence the minimalization problem of the formula (7) comes down to minimalization of the following sum

$$
\sum_{t=n}^{\infty}\left(\mathrm{E}\left(P_{t}-X_{t}\right)^{2}+\gamma^{2}\left(\mathrm{E}\left(X_{t} \mid \Theta\right)-\mathrm{E}\left(P_{t} \mid \Theta\right)\right)^{2}\right) p_{t},
$$

where we seek the optimum sequences $a_{i, t} \in \mathbb{R}$, for $p_{t}=\mathrm{P}(T \geq t)$. It is further simplified to $n$ minimalization problems. For all $t \geq n$ 
minimum should be held

$$
\min _{\left(a_{0, t}, \ldots, a_{t-1, t}\right) \in \mathbb{R}^{t}}\left(\mathrm{E}\left(P_{t}-X_{t}\right)^{2}+\gamma_{t}^{2} \mathrm{E}\left(\mathrm{E}\left(P_{t}-X_{t} \mid \Theta\right)\right)^{2}\right) .
$$

Above problem is one period case, which was solved in the section 1 . Thus the optimal stream of premiums is given by the formula

$$
P_{t}\left(\gamma_{t}\right)=z_{t}\left(\gamma_{t}\right) \bar{X}_{t-1}+\left(1-z_{t}\left(\gamma_{t}\right)\right) m, \quad t=n, n+1, \ldots, T,
$$

where

$$
z_{t}\left(\gamma_{t}\right)=\frac{a^{2}\left(1+\gamma_{t}^{2}\right) n}{a^{2}\left(1+\gamma_{t}^{2}\right) n+s^{2}} .
$$

The premium (8) satisfies the net premium principle, i.e., $\mathrm{E} P_{t}\left(\gamma_{t}\right)=$ $\mathrm{E} X_{t}$ for all $\gamma_{t} \geq 0$ and it can be presented as

$$
P_{t}\left(\gamma_{t}\right)=\left(1-\beta_{t}\left(\gamma_{t}\right)\right) \bar{X}_{t-1}+\beta_{t}\left(\gamma_{t}\right) P_{t}^{C r},
$$

where $P_{t}^{C r}$ is credibility premium (1) and

$$
\beta_{t}\left(\gamma_{t}\right)=\frac{1-z_{t}\left(\gamma_{t}\right)}{1-z_{t}(0)}
$$

where $0<\beta_{t}\left(\gamma_{t}\right) \leq 1$. Note that

$$
U_{t}(\theta):=\mathrm{E}\left(P_{t}\left(\gamma_{t}\right)-P_{t}^{C r} \mid \Theta=\theta\right)=\left(1-\beta_{t}\left(\gamma_{t}\right)\right)\left(1-z_{t}(0)\right)(\mu(\theta)-m) .
$$

Thus if insured person is a good client $(\mu(\theta)<m)$, then $U_{t}(\theta)<0$, and when bad $(\mu(\theta)>m)$, then $U_{t}(\theta)>0$. In comparison to credibility premium, good client pays on average less and bad client more. Furthermore, we have

$U_{t+1}(\theta)-U_{t}(\theta)=\frac{\left[\left(\beta_{t}-\beta_{t+1}\right)\left(s^{2}+t a^{2}\right)-\left(1-\beta_{t+1}\right) a^{2}\right] s^{2}}{\left(s^{2}+t a^{2}\right)\left(s^{2}+(t-1) a^{2}\right)}(\mu(\theta)-m)$,

where $\beta_{t}=\beta_{t}\left(\gamma_{t}\right)$. If sequence of fixed numbers $\left(\gamma_{t}\right)$ is set such as the stream of $\left(\beta_{t}\right)$ satisfies

$$
\left(\beta_{t}-\beta_{t+1}\right)\left(s^{2}+t a^{2}\right)>\left(1-\beta_{t+1}\right) a^{2},
$$

than the difference between average premium $P_{t}\left(\gamma_{t}\right)$ paid by good client and average premium $P_{t}^{C r}$ rises simultaneously with time.

The stream (8) is also optimal when $T$ is any type of stopping moment and minimalisation functions is given by formula

$$
J=\mathrm{E}\left[\sum_{t=n}^{T}\left(\mathrm{E}\left(P_{t}-X_{t}\right)^{2}+\gamma_{t}^{2} \mathrm{E}\left(\mathrm{E}\left(X_{t} \mid \Theta\right)-\mathrm{E}\left(P_{t} \mid \Theta\right)\right)^{2}\right) \mid T \geq n\right],
$$


because

$$
J=\sum_{t=n}^{\infty}\left[\mathrm{E}\left(P_{t}-X_{t}\right)^{2}+\gamma_{t}^{2} \mathrm{E}\left(\mathrm{E}\left(X_{t} \mid \Theta\right)-\mathrm{E}\left(P_{t} \mid \Theta\right)\right)^{2}\right] \mathrm{P}(T \geq t \mid T \geq n),
$$

but the stopping moment must be chosen in such a way that the probability $\mathrm{P}(T \geq t \mid T \geq n)$ is not a function of coefficients $a_{i, t}$. Examples of stopping moments are

1. $T_{1}=\inf \left\{t \geq n: X_{1}+\ldots+X_{t}>c_{t}\right\}$, where $c_{t}>0$ is any sequence of real numbers and $\inf \emptyset=\infty$. The interpretation of this example is a case when insurer does not renew the insurance contract because the client aggregate loss exceeds predefined thresholds $c_{t}$.

2. $T_{2}=\inf \left\{t \geq n: \max _{1 \leq n \leq t-1} X_{n}<c_{t} X_{t}\right\}$. The insurance company does not renew the insurance contract when extraordinary claim appears (a claim, which is multiple times bigger than the previous claims). Similar case is when $T_{2}^{\prime}=\min \left(t \geq n: X_{1} \leq c_{1}, \ldots, X_{t-1} \leq c_{t-1}, X_{t}>\right.$ $\left.c_{t}\right)$.

3. $T_{2}=\inf \left\{t \geq n: \rho_{t}\left(X_{1}, \ldots, X_{t}\right)>c_{t}\right\}$, where $\rho_{t}$ is any risk measure, i.e., $\rho_{t}=\sum_{i=1}^{t} \alpha_{i} X_{i: t}$, where $X_{i: t}$ is the $i$ th ranking statistic from sequence $X_{1}, \ldots, X_{t}$.

4. $T_{4}=\min \left\{T_{k}, T\right\}$, where $T_{k}, k=1,2,3$, are stopping moments defined in the previous points and $T$ is future expected long of life or the contract boundaries.

As far as we know, the first modifications of credibility premium in multiperiod model was proposed by Gajek et al. (2007). We will summarize results of this paper. Let $X_{-n}, \ldots, X_{-1}$ be losses occurred before moment 0 , in which insurance policy lasting $T$ years is written. The following premiums $P_{1}, \ldots, P_{T}$ cover losses described by random variables $X_{1}, \ldots, X_{T}$. It is assumed, that client can not resign from the contract. Thus the $T$ is fixed. Gajek et al. (2007) proposed following modification of credibility premium

(11) $P_{t}^{G M S}=\alpha_{t}\left(\sum_{i=1}^{t-1} X_{i}+(T-t+1) P_{t}^{C r}\right), \quad t=1,2, \ldots, T$,

where $\sum_{i=1}^{0} X_{i}=0$ and $\alpha_{t} \geq 0$ are numbers, which mimalize two distance functions. Furthermore

$$
P_{t}^{C r}=z_{t} \frac{X_{-n}+\ldots+X_{-1}+\sum_{i=1}^{t-1} X_{i}}{t-1+n}+\left(1-z_{t}\right) m
$$


where

$$
z_{t}=\frac{a^{2}(t-1+n)}{s^{2}+a^{2}(t-1+n)} .
$$

Note that the family of premiums (11) does not include every linear functions of $X_{1}, \ldots, X_{t-1}$. Authors presents analysis, which recommends use of sequence $\alpha_{t}=1 / T$. In that case the recommended premiums are equal to

$$
\widehat{P}_{t}^{G M S}=\frac{1}{T}\left(\sum_{i=1}^{t-1} X_{i}+(T-t+1) P_{t}^{C r}\right), \quad t=1,2, \ldots, T
$$

(see [4], pp.230-232). Further it is presented that the modified premiums are more adequate than credibility premiums

$$
U_{t}(\theta)=\mathrm{E}\left[\widehat{P}_{t}^{G M S}-P_{t}^{C r} \mid \Theta=\theta\right]=\frac{s^{2}(t-1)}{T\left(s^{2}+a^{2}(t-1+n)\right)}(\mu(\theta)-m) .
$$

Good clients pay on average less than in the case of basic credibility premium. Moreover for all $t<T$

$$
U_{t+1}(\theta)-U_{t}(\theta)=s^{2} T \frac{s^{2}+n a^{2}}{\left(s^{2}+(n+t) a^{2}\right)\left(s^{2}+(n+t-1) a^{2}\right)} \geq 0,
$$

which indicates that the surplus of good clients rise simultaneously with time. This property seems to be desired by good clients and in the opinion of the authors it will dispose potential clients to write the insurance policy. At the same time bad clients will prefer based credibility premium.

However calculations of premiums (12) points a problem. Let us assume, that the good client is considering to buy the $T$ year insurance contract. He believes that he is good, because $X_{-n}=\ldots=X_{-1}=0$, but he reckons with appearance of claim in the first period $X_{1}>0$, and later $X_{2}=\ldots=X_{T}=0$. Hence

$\widehat{P}_{1}^{G M S}=P_{1}^{C R}, \quad \widehat{P}_{2}^{G M S}=\frac{1}{T} X_{1}+\frac{T-1}{T} P_{1}^{C r}, \ldots, \widehat{P}_{T}^{G M S}=\frac{1}{T} X_{1}+\frac{1}{T} P_{T}^{C r}$,

thus sum of the whole stream of premiums is equal to

$$
\sum_{t=1}^{T} \widehat{P}_{t}^{G M S}=\frac{T-1}{T} X_{1}+P_{1}^{C r}+\frac{T-1}{T} P_{2}^{C r}+\ldots+\frac{1}{T} P_{T}^{C r} .
$$

In spite of the decrease of premiums, the total sum is approximated to the single claim $X_{1}$, thus the insurance policy does not provide necessary insurance coverage. 
Note that the problem appeared because in the first component of the sum (12) does not include the losses $X_{-n}, \ldots, X_{-1}$. We propose following the adjustment of the stream (12)

$$
\widehat{P}_{t}=\left(1-\beta_{t}\right) \frac{1}{t-1+n} \sum_{i=-n}^{t-1} X_{i}+\beta_{t} P_{t}^{C r},
$$

where

$$
\beta_{t}=\frac{T-t+1}{T}
$$

Note that the formula is held

$$
U_{t}(\theta)=\mathrm{E}\left[\widehat{P}_{t}-P_{t}^{C r} \mid \Theta=\theta\right]=\mathrm{E}\left[\widehat{P}_{t}^{G M S}-P_{t}^{C r} \mid \Theta=\theta\right]
$$

for all $t=1,2, \ldots, T$ and $\theta$. In other words the stream of premiums (13) satisfies the same properties of making distinction between good and bad clients. On the other hand from equations (13) and (10) it follows that the stream (13) minimalizes the sum

$$
\mathrm{E}\left[\sum_{t=1}^{T}\left(\left(P_{t}-X_{t}\right)^{2}+\gamma_{t}^{2}\left(\mathrm{E}\left(P_{t}-X_{t} \mid \Theta\right)\right)^{2}\right)\right],
$$

where

$$
P_{t}=a_{0, t}+\sum_{i=-n}^{-1} a_{i, t} X_{i, t}+\sum_{j=1}^{t-1} a_{j, t} X_{j, t}, \quad a_{i, j} \in \mathbb{R},
$$

and the $\gamma_{t}$ in (14) is given by

$$
\gamma_{t}^{2}=\frac{(t-1)\left(s^{2}+a^{2}(t-1+n)\right.}{(T-t+1) a^{2}(t-1+n)}, \quad t=1, \ldots, T .
$$

Stream $\widehat{P}_{t}$ satisfies the net premium principle $\mathrm{E} \widehat{P}_{t}=\mathrm{E} X_{t}, t=1, \ldots, T$, Hence a weak Axiom of Solvency is satisfied, i.e., $\mathrm{E} \sum_{s=1}^{t} \widehat{P}_{s} \geq \mathrm{E} \sum_{s=1}^{t} X_{s}$ for all $t$ and $\mathrm{E} \sum_{t=1}^{T} P_{t}=\mathrm{E} \sum_{t=1}^{T} X_{t}$ (see $\underline{\text { Gajek }}$ et al., 2007). Example 1 shows, the differences between (12) and (13).

Example 1. Let $T=5$ years, $n=10$ years, $X_{-10}=X_{-9}=\ldots, X_{-1}=$ $0, X_{1}=20000, X_{2}=\ldots=X_{5}=0$. The stream of premiums proposed by Gajek et al. (2007), formula (12), is

$$
\begin{gathered}
P_{1}^{G M S}=P_{1}^{C r}, \quad P_{2}^{G M S}=4000+\frac{4}{5} P_{1}^{C r}, \quad P_{3}^{G M S}=4000+\frac{3}{5} P_{1}^{C r}, \\
P_{4}^{G M S}=4000+\frac{2}{5} P_{1}^{C r}, \quad P_{5}^{G M S}=4000+\frac{1}{5} P_{1}^{C r} .
\end{gathered}
$$


Total sum of premiums during the 5 years is equal to $16000+3 P_{1}^{C r}$ when only one claim occurred 20000 . Using modified formula (13) we have

$$
\begin{gathered}
P_{1}=P_{1}^{C r}, \quad P_{2}=363+\frac{4}{5} P_{1}^{C r}, \quad P_{3}=666+\frac{3}{5} P_{1}^{C r}, \\
P_{4}=923+\frac{2}{5} P_{1}^{C r}, \quad P_{5}=1142+\frac{1}{5} P_{1}^{C r},
\end{gathered}
$$

and the sum is equal to $P_{1}+\ldots+P_{5}=3094+3 P_{1}^{C r}$.

\section{REFERENCES}

[1] Bowers N.L., Gerber H.U., Hickman J.C., Jones D.A., Nesbitt C.J. (1997) Actuarial Mathematics. The Society of Actuaries, 2nd Edition, Schaumburg, III.

[2] Bühlmann H. (1996), Mathematical Methods in Risk Theory. 2nd edition, Springer-Verlag, Berlin.

[3] Bühlmann H., Gisler A. (2005) A Course in Credibility Theory and its Applications, Springer 2005.

[4] Gajek, L., Miś, P., Słowińska, J. (2007) Optimal streams of premiums in multiperiod credibility models, Applicationes Mathematicae 34, 223-235.

[5] Gómez-Déniz E. (2008) A generalization of the credibility theory obtained by using the weighted balanced loss function. Insurance: Mathematics and Economics 42(2), 850-854.

[6] Jasiulewicz, H. (2005) Credibility Theory. AE, Wrocław (in Polish).

[7] Kim, J.H.T., Jeon, Y. (2013) Credibility theory based on trimming. Insurance: Mathematics and Economics 53(1), 36-47.

[8] Lau, J. W., Siu, T.K., Yang, H. (2006) On Bayesian mixture credibility. ASTIN Bulletin 36 (2), 573-588.

[9] Niemiro, W.. (2006) Bayesian prediction with an asymmetric criterion in a nonparametric model of insurance risk. Statistics 40(4), 353-363

[10] Pan, M., Wang, R., Wu, X. (2008) On the consistency of credibility premiums regarding Esscher principle. Insurance: Mathematics and Economics 42(1), 119-126.

[11] Payandeh Najafabadi, A. T. (2010) A new approach to the credibility formula. Insurance: Mathematics and Economics 46(2), 334-338.

[12] Payandeh Najafabadi, A. T., Hatami, H., Omidi Najafabadi, M. (2012) A maximum-entropy approach to the linear credibility formula. Insurance Mathematics and Economics 51(1), 216-221.

[13] Pitselis, G. (2013) Quantile credibility models. Mathematics and Economics 52(3), 477-489.

[14] Promislow, S.D., Young, V.R. (2000) Equity and exact credibility, ASTIN Bulletin 30, 3-11. 
[15] Wen, L., Wu, X., Zhou, X. (2009) The credibility premiums for models with dependence induced by common effects. Insurance: Mathematics and Economics 44, 19-25.

[16] Wen, L.M., Wang, W., Wang, J.L. (2011) The credibility premiums for exponential principle. Acta Mathematica Sinica, English Series, 27(11), 2217-2228. 